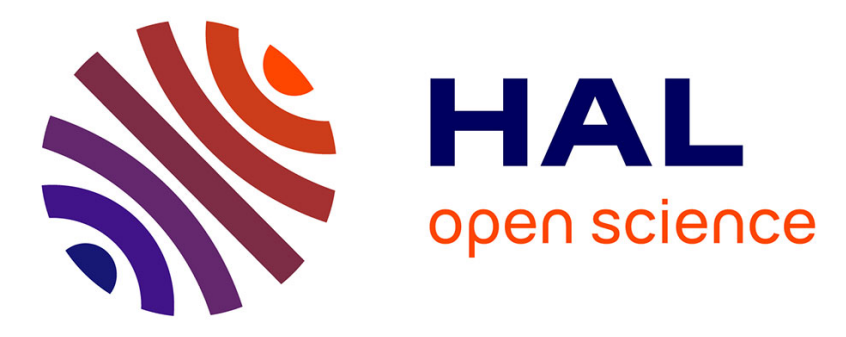

\title{
Hilbert Fractal Inspired Dipoles for B1+ Field Control in Ultra-High Field MRI
}

Tania S. Vergara Gomez, M. Dubois, K. Rustomji, E. Georget, T. Antonakakis, S. Rapacchi, F. Kober, S. Enoch, R. Abdeddaim

\section{- To cite this version:}

Tania S. Vergara Gomez, M. Dubois, K. Rustomji, E. Georget, T. Antonakakis, et al.. Hilbert Fractal Inspired Dipoles for B1+ Field Control in Ultra-High Field MRI. 2020 Fourteenth International Congress on Artificial Materials for Novel Wave Phenomena (Metamaterials), Sep 2020, New York City, United States. pp.327-329, 10.1109/Metamaterials49557.2020.9285012 . hal-03164033

\section{HAL Id: hal-03164033 https://hal.science/hal-03164033}

Submitted on 9 Mar 2021

HAL is a multi-disciplinary open access archive for the deposit and dissemination of scientific research documents, whether they are published or not. The documents may come from teaching and research institutions in France or abroad, or from public or private research centers.
L'archive ouverte pluridisciplinaire HAL, est destinée au dépôt et à la diffusion de documents scientifiques de niveau recherche, publiés ou non, émanant des établissements d'enseignement et de recherche français ou étrangers, des laboratoires publics ou privés. 


\title{
Hilbert Fractal Inspired Dipoles for $\mathrm{B}_{1}{ }^{+}$Field Control in Ultra-High Field MRI
}

\author{
$\underline{\text { T.S. Vergara Gomez }}{ }^{1,2}$, M. Dubois ${ }^{1,2}$, K. Rustomji ${ }^{3}$, E. Georget ${ }^{3}$, T. Antonakakis ${ }^{3}$, S. Rapacchi ${ }^{1}$, F. \\ Kober' $^{1}$, S. Enoch ${ }^{2}$, R. Abdeddaim ${ }^{2}$ \\ ${ }^{1}$ Aix Marseille Univ, CNRS, CRMBM, Marseille, France \\ ${ }^{2}$ Aix Marseille Univ, CNRS, Centrale Marseille, Institut Fresnel, Marseille, France \\ ${ }^{3}$ Multiwave Imaging, Marseille, France \\ tania-del-socorro.vergara-gomez@etu.univ-amu.fr
}

\begin{abstract}
Due to the reduction of wavelengths inside the human tissues at ultra-high field magnetic resonance imaging (MRI), head coils usually present $\mathrm{B}_{1}{ }^{+}$magnetic field inhomogeneities located at the temporal lobes of the brain. This problem has been effectively targeted by different approaches, with the downside that they can either decrease the $\mathrm{B}_{1}{ }^{+}$field in other important areas or be uncomfortable to the patient. Here, we present a new approach based on Hilbert fractal inspired dipoles. Our structures are able to improve simultaneously the $\mathrm{B}_{1}^{+}$field in each temporal lobe without diminishing the patients' comfort or the $\mathrm{B}_{1}^{+}$field in other parts of the brain.
\end{abstract}

\section{INTRODUCTION}

The constructive and destructive interferences between wavelengths at ultra-high magnetic field (7 Teslas, $300 \mathrm{MHz}$ ) in clinical magnetic resonance imaging (MRI) lead to head coils' $\mathrm{B}_{1}^{+}$magnetic field inhomogeneities in the temporal lobe regions. Approaches like high-permittivity dielectric pads [1]-[3] and coupled-wire metamaterials [4]-[5] have been employed to mitigate these effects. They present a powerful and controllable improvements of the $\mathrm{B}_{1}{ }^{+}$field inhomogeneities, but there remain some challenges to overcome.

The dielectric pads are based on the principle of displacement currents and are mainly composed of highpermittivity materials like calcium titanate $\left(\mathrm{CaTiO}_{3}\right)$ or barium titanate $\left(\mathrm{BaTiO}_{3}\right)$ mixed with water [1]-[3]. Their dimensions and permittivity can be optimized for a specific target. $\mathrm{BaTiO}_{3}$ pads with 250 relative permittivity and $10 \times 10 \times 1 \mathrm{~cm}^{3}$ volume are examples of pads use for head imaging at 7T. They are place directly in contact with the patient's head. Since these pads are bulky, they are limited by the patients' morphology and the head coils' space which can be reduced if they are equipped with a receive coil array. This adds constraints in the set up and reduction of the patient comfort. On the other hand, the metamaterial approach is based on the mode hybridization principle, using long resonant dipoles coupled into a specific geometry [4]-[5]. Designs such as the Hybridized Meta-Atom (HMA) provide stronger enhancement than dielectric pads since it is possible to control the coil-resonator coupling strength by adapting the length of the wires and the position with respect to the patient's head, giving more degrees of freedom. They can be placed away from the patient's head, but sometimes it can be hard to fit the resonator in the small and tight free space left in commercial MRI coils. HMA have strongly improved the $\mathrm{B}_{1}{ }^{+}$field at a single region of interest like one temporal lobe. However, maintaining such performance while targeting multiple areas by using several structures may be difficult.

Here, we propose compact, thin and flexible resonant meander dipoles based on a Hilbert fractal. The main concept behind these meander electric dipoles is to introduce small passive resonators free of lumped elements, able to resonate at $300 \mathrm{MHz}$ and to fit within a maximum of $10 \times 10 \mathrm{~cm}^{2}$ area. First, we investigated space filling structures based on Hilbert fractal [6]-[7]. Hilbert fractals end-feed have been used as monopoles [6]. In order to satisfy our geometrical constraints, we used two $3^{\text {rd }}$-order Hilbert monopoles in series to form our passive meander electric dipole. Akin to dielectric pads, the Hilbert like dipoles are placed close to the patient's head. Due to their sub millimeter thickness, they do not impact the patients' comfort. Our compact and flexible structures are able to improve the $\mathrm{B}_{1}{ }^{+}$field in each temporal lobe maintaining the transmit efficiency of the head coil elsewhere.

\section{MATERIAL AND METHODS}

Exhaustive numerical studies were carried out on CST Microwave studio to compare the effect on the 16-legs head coil's $\mathrm{B}_{1}{ }^{+}$field while using the original $3^{\text {rd }}$-order Hilbert fractal and our proposed meander dipole (Fig. 1). The head coil was fed by 2 ports in quadrature. A third port was placed on the structure used to check the 
resonance and the coupling with the head coil. This third port was later short-circuited to obtain $\mathrm{B}_{1}{ }^{+}$magnetic field maps with the structure inductively coupled to the main coil. We printed the copper meander dipoles on a 0.4-mm-thick FR-4 substrate to make them thin and flexible. They were tested using a 1Tx/32Rx proton head coil (Nova Medical, Wilmington, MA, USA) in a 7T Magnetom MRI scanner (Siemens Healthineers, Erlangen, Germany) using a specific anthropomorphic head phantom (SPEAG, Zurich, Switzerland) filled with a liquid with permittivity $\varepsilon=45.3$ and conductivity $\sigma=0.87 \mathrm{~S} / \mathrm{m}$. Using the turbo-flash XFL MRI sequence [8], we obtained $\mathrm{B}_{1}{ }^{+}$field distribution maps in sagittal and axial orientation, with the following parameters: repetition time $=20 \mathrm{~s}$, echo time $=1.4 \mathrm{~ms}$, flip angle $=8^{\circ}$, Shinnar-Le Roux minimum-phase saturation pulse $=90^{\circ}, \mathrm{FOV}=$ $256 \mathrm{~mm}$, matrix $=64 \times 64$ and 12 slices of $8 \mathrm{~mm}$ thickness (17 slices in axial orientation). All images were acquired using the same reference voltage of $260 \mathrm{~V}$. For a quantitative analysis, we computed relative $\mathrm{B}_{1}^{+}$field difference maps expressed in percentage.

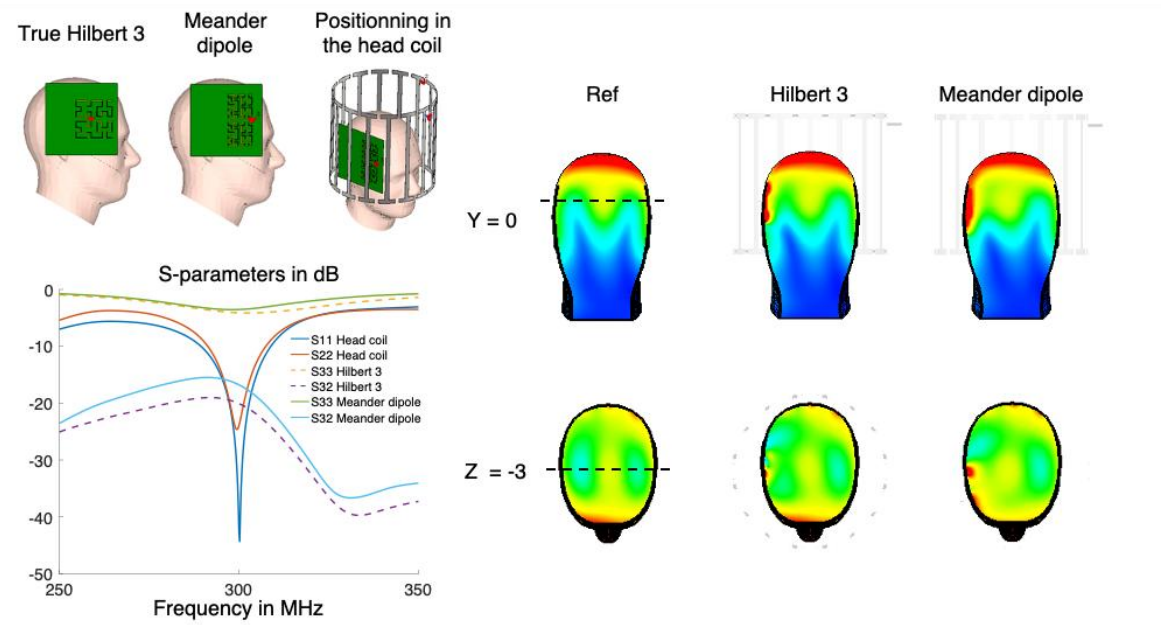

Fig. 1. Design and study of original Hilbert fractal and meander dipole. Top left, structures' design and position with respect to the head and the coil. Bottom left, S-parameters showing the coupling between the structures and the head coil. Right side, Amplitude of $\mathrm{B}_{1}{ }^{+}$field on $\mathrm{Y}$ direction at the center of the phantom and $\mathrm{Z}$ direction at $-3 \mathrm{~cm}$ below the center. The dash lines show the position where the slices were obtained, color scale from 0 to $0.5 \mu \mathrm{T}$.

\section{RESULTS}

According to the S-parameters in Fig. 1, both S33 parameters indicate that Hilbert fractal and the meander dipole resonate around $300 \mathrm{MHz}$. S31 and S32 coefficients characterize the coupling to each head coil ports. We only present $\mathrm{S} 32$ results as it is the dominant contribution. With the meander dipole we obtain a better coupling $(+3 \mathrm{~dB})$ to the head coil compared to the original Hilbert fractal. The right side of Fig. 1 shows the simulated $\mathrm{B}_{1}^{+}$ field maps obtained from a head coil alone (reference) and coupled with the Hilbert fractal and with the meander dipole. The reference present characteristic inhomogeneities (regions with low amplitude) at the temporal region of the brain. We can see that the Hilbert fractal has an effect only on the surface of the phantom. On the other hand, the meander dipole is able to enhance $\mathrm{B}_{1}{ }^{+}$field further in the phantom and on a larger area.

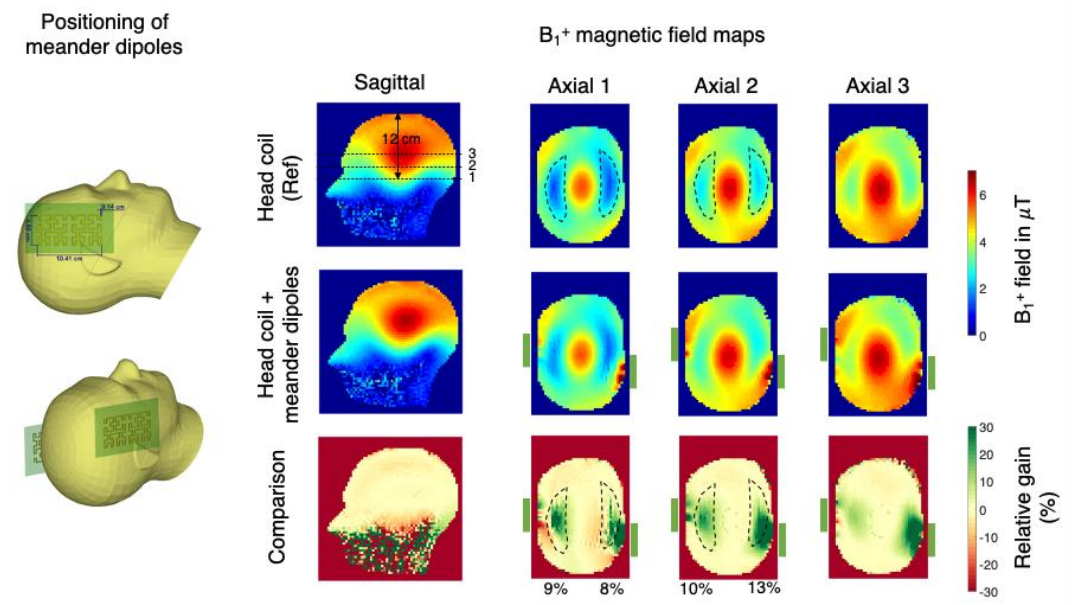


Fig. 2. Position of the meander dipoles (left) and $\mathrm{B}_{1}{ }^{+}$field distribution maps of the head coil alone (top right) and coupled with the resonant meander dipoles (middle right) in sagittal and axial orientations. The bottom right row shows the relative gain between meander dipoles and reference map.

The experimental results show the effect of the meander dipoles at different locations of the phantom head (Fig. 2). A central sagittal slice and three different axial slices of $\mathrm{B}_{1}{ }^{+}$field maps are presented. The green rectangles next to the axial slices represent schematically the position of the meander dipoles with respect to the phantom. The dashed lines on the sagittal slices show the different examined locations selected through the head. The first axial slice analyzed is located at $12 \mathrm{~cm}$ from the top of the head and the space between two consecutive axial slices is $24 \mathrm{~mm}$. At the bottom of Fig. 2, difference maps were calculated. We defined the temporal lobe ROIs from the reference scan. The values at the bottom of each figure are the mean values obtained inside each temporal lobe ROI. A significant increase of the $\mathrm{B}_{1}{ }^{+}$field can be seen when using the meander dipoles. A $30 \%$ gain was obtained around the area were the meander dipoles were placed, and $10 \%$ gain was obtained on average in the ROIs that denote the temporal lobe regions. Furthermore, the central sagittal slice and the lower axial slice illustrate that no loss is observed in other regions of the brain such as the cerebellum.

\section{DISCUSSION AND CONCLUSIONS}

By designing the passive meander dipoles based on a Hilbert's fractal we were able to obtain compact, thin and flexible resonant structures. We show in the first section that our passive meander dipole design outperforms the original Hilbert fractal structure. The original Hilbert fractal design mostly radiates a magnetic field normal to the fractal plane (similar to a loop). Since the main $\mathrm{B}_{1}{ }^{+}$magnetic field of the head coil is parallel to the fractal plane, this reduces the inductive coupling between them. Here, we proposed the meander dipole design based on two connected fractal patterns, obtaining a magnetic field polarization collinear to the main $\mathrm{B}_{1}{ }^{+}$field produced by the head coil. Thus, a better coupling (S32 coefficient) and a better $\mathrm{B}_{1}{ }^{+}$field enhancement can be obtained.

The concept was studied experimentally on a phantom head using two meander dipoles, one on each side of the head. Due to their design, the meander dipoles are quite sensitive to the position and orientation with respect to the head coil. Therefore, some tests were needed to find the most efficient position for each meander dipole, respecting the original asymmetry given by the reference $\mathrm{B}_{1}{ }^{+}$field distribution maps. This is the reason for the asymmetric position of the meander dipoles with respect to the head. The experimental results show a strong correlation with the numerical studies. The presence of meander dipoles led to up to $30 \%$ gain in $\mathrm{B}_{1}{ }^{+}$field amplitude, with an average gain of $10 \%$ within both temporal lobes for the same input voltage (Fig. 2). This can be interpreted as a large increase in transmit efficiency. Furthermore, the field in the remaining brain volume remains almost intact. This last effect is particularly important for applications involving regions like the cerebellum, which usually exhibits field losses when other approaches are used. In our case, most of the loss seems to be directed towards the lower part of the head, close to the head coil's lower edge (Fig. 2).

The compact and flexible design of our meander dipoles make them fit easily between the phantom and the receive array of the head coil. Hence, our approach promises better performance and patient comfort over conventional approaches with dielectric pads or coupled-wire metamaterials.

\section{ACKNOWLEDGEMENTS}

This work has received funding from the European Union Horizon 2020 Research and Innovation program under Grant Agreement No. 736937.

\section{REFERENCES}

[1] Q.X. Yang, W. Mao, J. Wang, M.B. Smith, H. Lei, X. Zhang, et al, "Manipulation of image intensity distribution at 7.0 T: passive B1+ shimming and focusing with dielectric materials," J. Magn Reson., vol. 24, p. 197-202, 2006.

[2] A. G. Webb, "Dielectric materials in magnetic resonance," Concepts Magn. Reson. Part A, vol. 38A, p. $148,2011$.

[3] A. L. Neves, L. Leroi, Z. Raolison, N. Cochinaire, T. Letertre, et al, "Compressed perovskite aqueous mixtures near their phase transitions show very high permittivities: New prospects for high-field MRI dielectric shimming," Magn. Reson. Med., vol. 79, p. 1753, 2018.

[4] M. Dubois, L. Leroi, Z. Raolison, R. Abdeddaim, T. Antonakakis, et al, "Kerker Effect in Ultrahigh-Field Magnetic Resonance Imaging," Phys. Rev. X, vol. 8, p. 031083, 2018.

[5] M. Dubois, L. Leroi, Z. Raolison, R. Abdeddaim, T. Antonakakis, et al, "Enhancement of transmit and receive efficiencies with hybridized meta-atom in 7T head coil," Proc. Intl. Soc. Mag. Reson. Med., vol. 27, p. 1552, 2019.

[6] J. Anguera, C. Puente, E. Martinez, E. Rozan, "The fractal Hilbert monopole: A two-dimensional wire." Microwave and Optical technology letters, vol. 36.2, p. 102-104, 2003.

[7] N. Engheta, W.Z. Richard, "Metamaterials: physics and engineering explorations" John Wiley \& Sons, 2006.

[8] A. Amadon, F. Mauconduit, A. Vignaud, and N. Boulant, "Slice profile corrections in the XFL (magnetization-prepared turbo-FLASH) B1-mapping sequence," Proc. Intl. Soc. Mag. Reson. Med., vol.23, p. 2377, 2015. 